\title{
FINITE-RANK INTERMEDIATE HANKEL OPERATORS ON THE BERGMAN SPACE
}

\author{
TAKAHIKO NAKAZI and TOMOKO OSAWA
}

(Received 11 January 1998)

\begin{abstract}
Let $L^{2}=L^{2}(D, r d r d \theta / \pi)$ be the Lebesgue space on the open unit disc and let $L_{a}^{2}=L^{2} \cap \mathscr{H o l}(D)$ be the Bergman space. Let $P$ be the orthogonal projection of $L^{2}$ onto $L_{a}^{2}$ and let $Q$ be the orthogonal projection onto $\bar{L}_{a, 0}^{2}=\left\{g \in L^{2} ; \bar{g} \in L_{a}^{2}, g(0)=0\right\}$. Then $I-P \geq Q$. The big Hankel operator and the small Hankel operator on $L_{a}^{2}$ are defined as: for $\phi$ in $L^{\infty}, H_{\phi}^{\mathrm{big}}(f)=(I-P)(\phi f)$ and $H_{\phi}^{\text {small }}(f)=Q(\phi f)\left(f \in L_{a}^{2}\right)$. In this paper, the finite-rank intermediate Hankel operators between $H_{\phi}^{\text {big }}$ and $H_{\phi}^{\text {small }}$ are studied. We are working on the more general space, that is, the weighted Bergman space.
\end{abstract}

2000 Mathematics Subject Classification. Primary 47B35.

1. Introduction. Let $D$ be the open unit disc in $\mathbb{C}$ and let $d \mu$ be the finite positive Borel measure on $D$. Let $L^{2}=L^{2}(\mu)=L^{2}(D, d \mu)$ and $\mathscr{H o l}(D)$ be the set of all holomorphic functions on $D$. The weighted Bergman space $L_{a}^{2}=L_{a}^{2}(\mu)$ is the intersection of $L^{2}$ and $\mathscr{H} o l(D)$. In general, $L_{a}^{2}$ is not closed. In [6, Theorem 8], when $(\operatorname{supp} \mu) \cap D$ is a uniqueness set for $\mathscr{H} o l(D)$, the first author and M. Yamada gave a necessary and sufficient condition for that $L_{a}^{2}$ is closed. Throughout this paper, we assume that $L_{a}^{2}$ is closed. When $d \mu=r d r d \theta / \pi, L_{a}^{2}$ is the usual Bergman space.

For $\mu$ such that $L_{a}^{2}(\mu)$ is closed, when $\mu$ is the closed subspace of $L^{2}(\mu)$ and $z \mu \subseteq M$, $M$ is called an invariant subspace. Suppose that $M \supseteq z L_{a}^{2} . P^{\mu}$ denotes the orthogonal projection from $L^{2}$ onto $M$. For $\phi$ in $L^{\infty}=L^{\infty}(\mu)=L^{\infty}(D, d \mu)$, the intermediate Hankel operator $H_{\phi}^{\mu}$ is defined by

$$
H_{\phi}^{\mu} f=\left(I-P^{M}\right)(\phi f) \quad\left(f \in L_{a}^{2}\right) .
$$

When $\mathcal{M}=L_{a}^{2}, H_{\phi}^{\mathcal{M}}$ is called a big Hankel operator $H_{\phi}^{\text {big }}$ and when $\mathcal{M}=\left(\bar{z} \bar{L}_{a}^{2}\right)^{\perp}, H_{\phi}^{\mathcal{M}}$ is called a small Hankel operator $H_{\phi}^{\text {small }}$. Note that $H_{\phi}^{\mu}$ is called a little Hankel operator when $M=\left(\bar{L}_{a}^{2}\right)^{\perp}$.

For arbitrary symbol $\phi$ in $L^{\infty}$, in the case of $d \mu=r d r d \theta / \pi$, both $H_{\phi}^{\text {big }}$ and $H_{\phi}^{\text {small }}$ were studied when they are compact operators or Schatten class operators (see [12]). However it seems to have not been studied when they are finite-rank operators. When $\bar{\phi}$ is in $L_{a}^{2}$, it is known (see [12, page 155]) that if $H_{\phi}^{\text {big }}$ is a finite-rank operator, then $H_{\phi}^{\text {big }}=0$ and if $\bar{\phi}$ is a polynomial, then $H_{\phi}^{\text {small }}$ is a finite-rank operator. In this paper, for arbitrary symbol $\phi$ in $L^{\infty}$ we show that if $H_{\phi}^{\text {big }}$ is a finite-rank operator, then $H_{\phi}^{\text {big }}=0$, and we study when $H_{\phi}^{\text {small }}$ is a finite-rank operator. In fact, we study such problems for the intermediate Hankel operators $H_{\phi}^{\mu}$ on the weighted Bergman space $L_{a}^{2}(\mu)$. 
In $[2,7,9,10]$, intermediate Hankel operators were studied in special weights, $d \mu=$ $(\alpha+1)\left(1-r^{2}\right)^{\alpha} r d r d \theta / \pi$ for $-1<\alpha<\infty$. In particular, Strouse [9] studied finite-rank intermediate Hankel operators.

Let $d \mu=d \sigma(r) d \theta$ be a Borel measure on $D$, where $d \sigma(r)$ is a positive measure on $[0,1)$ with $d \sigma([0,1))=1 / 2 \pi$ and $d \theta$ is the Lebesgue measure on $\partial D . L_{a}^{2}(\mu)$ is closed if $d \sigma([t, 1))>0$ for any $t>0$ (see [6]). For this type measures, it is possible to study more precisely the intermediate Hankel operators. In fact, $L^{2}$ has the following orthogonal decomposition:

$$
L^{2}=\sum_{j=-\infty}^{\infty} \oplus \mathscr{L}^{2} e^{i j \theta},
$$

where $\mathscr{L}^{2}=L^{2}(d \sigma)=L^{2}([0,1), d \sigma)$. Set

$$
\mathbf{H}^{2}=\sum_{j=0}^{\infty} \oplus \mathscr{L}^{2} e^{i j \theta},
$$

then $L_{a}^{2} \subset \mathbf{H}^{2} \subset\left(\bar{z} \bar{L}_{a}^{2}\right)^{\perp}$ and $L^{2}=\mathbf{H}^{2} \oplus e^{-i \theta} \overline{\mathbf{H}}^{2}$. If $\boldsymbol{M}=\mathbf{H}^{2}$, it is easy comparatively to determine finite-rank Hankel operators $H_{\phi}^{\mu}$ and we can do it completely in Section 5. We can expect that $H_{\phi}^{\mu}$ is close to $H_{\phi}^{\text {big }}$ in case $\mu \subseteq \mathbf{H}^{2}$ (see Section 5) and $H_{\phi}^{\mu}$ is close to $H_{\phi}^{\text {small }}$ in case $\mathcal{M} \mathbf{H}^{2}$ (see Section 6).

In Section 2, we describe an invariant subspace in $L_{a}^{2}$ whose codimension is of finite. Moreover we show that there does not exist an invariant subspace which contains $L_{a}^{2}$ properly and in which $L_{a}^{2}$ is of finite codimension. We also give a lot of examples of invariant subspaces which contain $L_{a}^{2}$ and in which Hankel operators are studied in this paper. In Section 3, we describe finite-rank intermediate Hankel operators for arbitrary measure $\mu$ such that $L_{a}^{2}(\mu)$ is closed. Moreover, we show that there does not exist any nonzero finite-rank Hankel operators $H_{\phi}^{\text {big }}$ and there exists a nonzero finite-rank Hankel operator $H_{\phi}^{\text {small }}$. In fact, we give two necessary and sufficient conditions for that if $H_{\phi}^{\mu}$ is of finite rank $\leq \ell$, then $H_{\phi}^{\mu}=0$. In Sections 3, 4, and 5, we use the Fourier coefficients $\left\{\mathcal{M}_{j}\right\}_{j=-\infty}^{\infty}$ of $\mathcal{M}$ and so we assume $d \mu=d \sigma(r) d \theta$. Using the Fourier coefficients of $\phi$ and $\mathcal{M}$, we give a necessary and sufficient condition for that $H_{\phi}^{\mu}$ is of finite rank $\leq \ell$. Assuming that $\phi$ is a harmonic function, we can get a

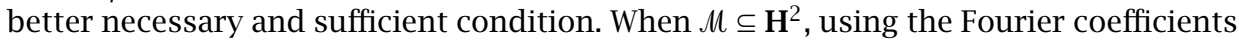
$\left\{\mu_{j}\right\}_{j=-\infty}^{\infty}$, we give a necessary condition and a sufficient condition for that if $H_{\phi}^{\mu}$ is of finite rank $\leq \ell$, then $H_{\phi}^{\mu}=0$. Two conditions are very similar but are a little different. Applications are given to examples in Section 2.

2. Invariant subspaces. In this section, we assume that $d \mu=d \sigma(r) d \theta$ and $d \sigma([t, 1))>0$ for any $t>0$, except Propositions 2.1 and 2.2. For our purpose, the invariant subspace $\mathcal{M}$ must contain $z L_{a}^{2}$ but $\operatorname{ker} H_{\phi}^{\mu}$ is an invariant subspace in $L_{a}^{2}$. If $H_{\phi}^{\mu}$ is of finite rank, then the codimension of $\operatorname{ker} H_{\phi}^{\mu}$ in $L_{a}^{2}$ is finite. In order to study finite-rank intermediate Hankel operators, we need the generalization of a result of Axler and Bourdon [1] which determines finite codimensional invariant subspaces in $L_{a}^{2}$ when $d \mu=r d r d \theta / \pi$. In Propositions 2.1 and 2.2, the measure $\mu$ is an arbitrary finite positive Borel measure such that $L_{a}^{2}$ is closed and $(\operatorname{supp} \mu) \cap D$ is a uniqueness set for $\mathscr{H} o l(D)$. Since $\mathbf{H}^{2} \cap L^{\infty}$ is an extended weak-* Dirichlet algebra in $L^{\infty}$, 
Proposition 2.3 is a corollary of [4, Theorem 1]. We will give several examples of invariant subspaces which contain $z L_{a}^{2}$.

Proposition 2.1. Suppose $M$ is an invariant subspace in $L_{a}^{2}$ and $\ell$ is a positive integer. The codimension of $\mathcal{M}$ in $L_{a}^{2}$ is $\ell$, if and only if $\mathcal{M}=q L_{a}^{2}$, where $q=\prod_{j=1}^{\ell}\left(z-a_{j}\right)$ and $a_{j} \in D(1 \leq j \leq \ell)$.

Proof. The proof is almost parallel to that in [1, Theorem 1]. We will give a sketch of it. Suppose $\mathcal{M}^{\perp}=L_{a}^{2} \ominus \mathcal{M}$ and $\operatorname{dim} \mathcal{M}^{\perp}=\ell$. Put

$$
S_{z} f=P(z f) \quad\left(f \in \mathcal{M}^{\perp}\right),
$$

where $P$ is an orthogonal projection. Since $\ell<\infty$, there exists an analytic polynomial $b$ such that $b\left(S_{z}\right)=S_{b(z)}=0$ and the degree of $b$ is less than or equal to $\ell$. Hence $b \mathcal{M}^{\perp} \subseteq \mathcal{M}$ and so $b L_{a}^{2} \subseteq \mathcal{M}$. We show that the zeros of $b$ are only in $D$ and the degree of $b=\ell$. Then $M=b L_{a}^{2}$. It is clear that the degree of $b=\ell$. In this direction, we did not need the condition such that $(\operatorname{supp} \mu) \cap D$ is a uniqueness set.

If $a \notin D,(z-a) L_{a}^{2}$ is dense in $L_{a}^{2}$. Assuming $a \geq 1$ and so $a=1$ without a loss of generality, if $\varepsilon>0$, then $(z-1) L_{a}^{2}=(z-1)\{z-(1+\varepsilon)\}^{-1} L_{a}^{2}$. For any $f \in L_{a}^{2}$, it is easy to see that

$$
\int_{D}\left|\frac{z-1}{z-(1+\varepsilon)} f-f\right|^{2} d \mu \longrightarrow 0 \quad(\varepsilon \rightarrow 0) .
$$

This implies that $(z-1) L_{a}^{2}$ is dense in $L_{a}^{2}$. Thus all zeros of $b$ must be in $D$. The "if" part is clear because any point $a \in D$ gives a bounded evaluation functional. Here we used the condition such that ( $\operatorname{supp} \mu) \cap D$ is a uniqueness set (see [6, (1) of Theorem 8]).

Proposition 2.2. Suppose that $(z-a)^{-1}$ does not belong to $L^{2}$ for each $a \in D$. If $M$ is an invariant subspace which contains $L_{a}^{2}$ properly, then the codimension of $L_{a}^{2}$ in $M$ is infinite.

Proof. If $\operatorname{dim} M \ominus L_{a}^{2}=\ell<\infty$, by the proof of Proposition 2.1, there exists a polynomial $b=\prod_{j=1}^{\ell}\left(z-a_{j}\right)$ such that $b \mu \subseteq L_{a}^{2}$ and $a_{j} \in D(1 \leq j \leq \ell)$. Hence there exists a function $\phi$ in $\mathcal{M}$ such that $\phi \notin L_{a}^{2}$ and $g=b \phi \in L_{a}^{2}$. If $g\left(a_{k}\right) \neq 0$ for some $k$, then $g /\left(z-a_{k}\right)=\phi \prod_{j \neq k}\left(z-a_{j}\right)$ cannot belong to $L^{2}$ because $\left(z-a_{k}\right)^{-1} \notin L^{2}$. Hence $g\left(a_{j}\right)=0$ for any $j$. By [6, the proof in (1) of Theorem 8], $g \in b L_{a}^{2}$ and so $\phi=g / b$ belongs to $L_{a}^{2}$. This contradiction implies that $\operatorname{dim} M \ominus L_{a}^{2}=\infty$.

For an invariant subspace $\mathcal{M}$, set

$$
\mathcal{M}_{j}=\left\{f_{j} \in \mathscr{L}^{2} ; f \in \mathcal{M}, f(z)=\sum_{j=-\infty}^{\infty} f_{j}(r) e^{i j \theta}\right\} .
$$

Then $\mu_{j}$ is a subspace in $\mathscr{L}^{2}, r M_{j} \subseteq M_{j+1}$ and hence $\operatorname{dim} M_{j+1} \geq \operatorname{dim} M_{j}$. We call $\left\{\mu_{j}\right\}_{j=-\infty}^{\infty}$ the Fourier coefficients of $\mu_{.} \mu_{j} e^{i j \theta}$ may not belong to $\mu$. If $\mu_{j} e^{i j \theta}$ belongs to $\mu$ for any $j$, then $\mu$ has the following decomposition:

$$
\mathcal{M}=\sum_{j=-\infty}^{\infty} \oplus \mathcal{M}_{j} e^{i j \theta} .
$$


This decomposition is called the Fourier decomposition of $\mu$. In general, $\mu$ does not have the Fourier decomposition but we can get an extension $\tilde{M}$ of $\mu$ which has the following Fourier decomposition:

$$
\tilde{M}=\sum_{j=-\infty}^{\infty} \oplus\left(\text { closure of } \mu_{j}\right) e^{i j \theta} .
$$

Proposition 2.3. If $\mathcal{M}$ is an invariant subspace which contains $L_{a}^{2}$ and $e^{i \theta} \mathcal{M} \subseteq \mathcal{M}$, then $\mathcal{M}=\chi_{E} \bar{q} \mathbf{H}^{2} \oplus \chi_{E^{c}} L^{2}$, where $\chi_{E}$ is a characteristic function in $\mathscr{L}^{2}$ and $q$ is a unimodular function in $\mathbf{H}^{2}$. Hence $\mathcal{M} \mathbf{H}^{2}$. If $\bigcap_{j=0}^{\infty} e^{i j \theta} \mathcal{M}=\{0\}$, then $\mathcal{M}=\bar{q} \mathbf{H}^{2}$.

Proof. Suppose $S_{0}=M \ominus e^{i \theta} \mathcal{M}$, then $\mathcal{M}=\left(\sum_{j=0}^{\infty} \oplus S_{0} e^{i j \theta}\right) \oplus \mathcal{M}_{-\infty}$, where $\mathcal{M}_{-\infty}=$ $\bigcap_{j=0}^{\infty} e^{i j \theta} \mathcal{M}$, and $r S_{0} \subset S_{0}$ because $r \mu_{j} \subseteq \mathcal{M}_{j+1}$. It is well known that $\mathcal{M}_{-\infty}=\chi_{G} L^{2}$ for a characteristic function $\chi_{F}$ of some measurable subset in $D$. Put $E=G^{c}$ then there exists a function $f$ in $S_{0}$ such that

$$
|f|>0 \quad \text { on } E \quad \text { and } \quad f=0 \quad \text { on } F .
$$

Since $f$ is orthogonal to $f e^{i j \theta}$ for all $j \geq 0 .|f|^{2}$ belongs to $\mathscr{L}^{1}=L^{1}(d \sigma)=L^{1}([0,1), d \sigma)$ and so $|f|$ belongs to $\mathscr{L}^{2}$. Hence $\chi_{E}$ belongs to $\mathscr{L}^{2}$. Set

$$
F\left(r e^{i \theta}\right)= \begin{cases}\frac{f\left(r e^{i \theta}\right)}{\left|f\left(r e^{i \theta}\right)\right|} & \text { if } f \neq 0, \\ 1 & \text { if } f=0,\end{cases}
$$

then $F$ is a unimodular function in $L^{2}$. Since $r S_{0} \subseteq S_{0}$, we can show that $\chi_{E} F$ belongs to $S_{0}$ and so $S_{0}=\chi_{E} F \mathscr{L}^{2}$. Hence $\mu \ominus \mu_{\infty}=\chi_{E} F \mathbf{H}^{2}$. Since $1 \in \mathcal{M}, \chi_{E} \bar{F} \in \mathbf{H}^{2}$ and $q=\bar{F} \in \mathbf{H}^{2}$,

EXAMPLE 2.4. (i) For $0<\beta<1$, put

$$
T_{\beta}=\overline{\operatorname{span}}\left\{z^{n} \bar{z}^{m} ; \beta n \geq m \geq 0\right\} .
$$

Then $T_{\beta}$ is an invariant subspace and $T_{\beta} \supseteq L_{a}^{2}$. Put $T_{\beta}=L_{a}^{2}$ for $\beta=0$ and $T_{\beta}=\mathbf{H}^{2}$ for $\beta=1$. In general, $L_{a}^{2} \subseteq T_{\beta} \subseteq \mathbf{H}^{2}$ and $T_{\beta}(0 \leq \beta<1)$ has the following Fourier decomposition:

$$
T_{\beta}=\sum_{j=0}^{\infty} \oplus\left(T_{\beta}\right)_{j} e^{i j \theta},
$$

where $\left(T_{\beta}\right)_{j}=\overline{\operatorname{span}}\left\{r^{j} p_{j}\left(r^{2}\right) ; \quad p_{j}\right.$ is a polynomial of degree at most $\left.\beta j /(1-\beta)\right\}$. Janson and Rochberg [2] studied $H_{\phi}^{M}$ when $\mathcal{M}=\left(\bar{T}_{\beta}\right)^{\perp}$. Then $\left(\bar{T}_{\beta}\right)^{\perp}=e^{i \theta} \mathbf{H}^{2} \oplus \sum_{j=0}^{\infty} \oplus$ $\left\{\mathscr{L}^{2} \ominus\left(\bar{T}_{\beta}\right)_{j}\right\} e^{-i j \theta}$.

(ii) For $k \geq 0$, put $E^{k}=\overline{\operatorname{span}}\left\{z^{m} \bar{z}^{n} ; m=0,1, \ldots, k ; n=m, m+1, \ldots\right\} . \bar{E}^{k}$ is an invariant subspace and $L_{a}^{2} \subseteq \bar{E}^{k} \subseteq \mathbf{H}^{2} . \bar{E}^{k}$ has the following Fourier decomposition:

$$
\bar{E}^{k}=\sum_{j=0}^{\infty} \oplus\left(\bar{E}^{k}\right)_{j} e^{i j \theta},
$$

where $\left(\bar{E}^{k}\right)_{j}=\operatorname{span}\left\{r^{j}, \ldots, r^{j+2 k}\right\}$. Strouse [9] studied $H_{\phi}^{\mu}$ when $\mathcal{M}=\left(E^{k}\right)^{\perp}$. Then $\left(E^{k}\right)^{\perp}=e^{i \theta} \mathbf{H}^{2} \oplus \sum_{j=0}^{\infty} \oplus\left\{\mathscr{L}^{2} \ominus\left(E^{k}\right)_{j}\right\} e^{-i j \theta}$. 
(iii) Fix a polynomial $p$ of degree $k$, that is, $p=\sum_{j=0}^{k} a_{j} z^{j}$. Put

$$
\begin{aligned}
Y(p) & =\overline{\operatorname{span}}\left\{z^{n}, z^{m} \bar{p} ; n \geq 0, m \geq 0\right\}, \\
Y^{k} & =\overline{\operatorname{span}}\left\{z^{\ell} \bar{z}^{j} ; \ell \geq 0,0 \leq j \leq k\right\} .
\end{aligned}
$$

Both $Y(p)$ and $Y^{k}$ are invariant subspaces and $L_{a}^{2} \subseteq Y(p) \subseteq Y^{k}$, and $Y^{k}$ has the following Fourier decomposition:

$$
Y^{k}=\sum_{j=-k}^{\infty} \oplus\left(Y^{k}\right)_{j} e^{i j \theta}
$$

where $Y_{0}^{k}=\operatorname{span}\left\{1, r^{2}, \ldots, r^{2 k}\right\}$ and $\left(Y^{k}\right)_{j}=r^{j}\left(Y_{0}^{k}\right)$ for $j \geq 0$, and $\left(Y^{k}\right)_{-j}=$ $\operatorname{span}\left\{r^{2 \ell-j} ; j \leq \ell \leq k\right\}$ for $1 \leq j \leq k .(Y(p))_{j} \subseteq\left(Y^{k}\right)_{j}$ for any $j$ but $Y(p)$ does not have a Fourier decomposition. If $a_{j} \neq 0$ for $1 \leq j \leq k,(Y(p))_{j}=\left(Y^{k}\right)_{j}$ for any $j$ and so $\tilde{Y}(p)=Y^{k}$. Peng, Rochberg, and Wu [7] and Wang and Wu [10] studied $H_{\phi}^{\mu}$ when $\mathcal{M}=\left(\bar{Y}^{k}\right)^{\perp}$. In general, we can define $Y(g)$ for any function $g$ in $L^{2}$. Usually, $Y(g)$ does not have the Fourier decomposition.

(iv) For a unimodular function $q$ in $\mathbf{H}^{2}$, put $\mu=\bar{q} \mathbf{H}^{2}$. Then $\mu$ is an invariant subspace which contains $\mathbf{H}^{2}$. In general, $\bar{q} \mathbf{H}^{2}$ may not have the Fourier decomposition but for $q=e^{i \ell \theta}$, for some $\ell \geq 0$,

$$
M=\sum_{j=-\ell}^{\infty} \oplus \mathscr{L}^{2} e^{i j \theta} .
$$

There are a lot of invariant subspaces between $\mathbf{H}^{2}$ and $e^{-i \ell \theta} \mathbf{H}^{2}$ even if $\ell=1$.

(v) For arbitrary closed subspaces $S$ in $\mathscr{L}^{2}$, put $\mathcal{M}=\mathbf{H}^{2} \oplus S e^{-i \theta}$. Then $M$ is an invariant subspace between $\mathbf{H}^{2}$ and $e^{-i \theta} \mathbf{H}^{2}$.

3. Kronecker's theorem. In this section, the measure $\mu$ is an arbitrary finite positive Borel measure such that $L_{a}^{2}$ is closed. We will write

$$
\mathcal{M}^{\infty}=\mathcal{M} \cap L^{\infty}
$$

and, for each positive integer $\ell$,

$$
\mathcal{M}^{\infty, \ell}=\left\{\phi \in L^{\infty} ; \phi(z)=g(z) \prod_{j=1}^{\ell}\left(z-a_{j}\right)^{-1} \text { a.e. } \mu \text { on } D, g \in \mathcal{M}^{\infty} \text { and } a_{1}, \ldots, a_{\ell} \in D\right\} .
$$

Then $\mu^{\infty} \subseteq \mu^{\infty, 1} \subseteq M^{\infty, 2} \subseteq \cdots$.

Kronecker (cf. [11, page 210]) described finite-rank Hankel operators on the Hardy space. Theorem 3.1 describes finite-rank intermediate Hankel operators on the (weighted) Bergman space. However the situation is very different from that of Kronecker because $\mathcal{M}^{\infty}=\mathcal{M}^{\infty, \ell}$ may happen for some $\ell>0$. See Corollaries 3.3 and 3.4.

THEOREM 3.1. Suppose $\mathcal{M}$ is an invariant subspace which contains $z L_{a}^{2}$, and $\phi$ is a function in $L^{\infty} . H_{\phi}^{\mathcal{M}}$ is of finite rank $\leq \ell$ if and only if $\phi$ belongs to $\mathcal{M}^{\infty, \ell}$.

Proof. Note that $\operatorname{ker} H_{\phi}^{\mathcal{M}}=\left\{f \in L_{a}^{2} ; \phi f \in \mathcal{M}\right\}$. Since $\mathcal{M}$ is an invariant subspace, ker $H_{\phi}^{\mathcal{M}}$ is also an invariant subspace. Proposition 2.1 implies the theorem. 
THEOREM 3.2. Suppose $M$ is an invariant subspace which contains $L_{a}^{2}$, and $\phi$ is a function in $L^{\infty}$. Then the following are equivalent:

(1) If $H_{\phi}^{\mu}$ is of finite rank, then $H_{\phi}^{\mu}=0$.

(2) $\mu^{\infty}=\mu^{\infty, \ell}$ for any $\ell>0$.

(3) If $g \in \mathcal{M}^{\infty}, a \in D$ and $(g(z)-g(a)) /(z-a) \in L^{\infty}$, then $(g(z)-g(a)) /(z-a)$ belongs to $M^{\infty}$.

(4) If $\mathcal{M}^{\prime}$ is an invariant subspace and $\left(\mathcal{M}^{\prime}\right)^{\infty} \supsetneq \mathcal{M}^{\infty}$, then there does not exist a nonzero polynomial $b$ such that $b\left(\mathcal{M}^{\prime}\right)^{\infty} \subseteq \mathcal{M}^{\infty}$.

Proof. By Theorem 3.1, (1) $\Leftrightarrow(2)$ is clear.

(1) $\Rightarrow$ (3). If there exists $g \in \mathcal{M}^{\infty}$ such that $(g-g(a)) /(z-a) \in L^{\infty}$ does not belong to $\mathcal{M}^{\infty}$, put $\phi=(g-g(a)) /(z-a)$, then $H_{\phi}^{\mu}$ is of rank 1 and $H_{\phi}^{\mu} \neq 0$.

(3) $\Rightarrow$ (4). If (4) is not true, there exists $\psi$ such that $\psi \notin \mathcal{M}^{\infty}, \psi \in\left(\mathcal{M}^{\prime}\right)^{\infty}$ and $b \psi \in \mathcal{M}^{\infty}$ for some polynomial: $b=\prod_{j=1}^{\ell}\left(z-a_{j}\right)$ and $a_{j} \in D(1 \leq j \leq \ell<\infty)$. We may assume that $\phi=\psi \prod_{j=1}^{\ell-1}\left(z-a_{j}\right) \notin \mathcal{M}^{\infty}$ and $g=\left(z-a_{\ell}\right) \phi \in \mathcal{M}^{\infty}$. Then

$$
\frac{g-g\left(a_{\ell}\right)}{z-a_{\ell}}=\phi \in L^{\infty}, \quad \phi \notin \mathcal{M}^{\infty}
$$

(4) $\Rightarrow(1)$. By Theorem 3.1, if $H_{\phi}^{\mathcal{M}}$ is of finite rank $\leq \ell$, then $\phi \in \mathcal{M}^{\infty, \ell}$. If $\phi \notin \mathcal{M}^{\infty}$, suppose $\mathcal{M}^{\prime}$ is an invariant subspace generated by $\phi$ and $\mathcal{M}$, then $\left(\mathcal{M}^{\prime}\right)^{\infty} \supsetneq \mathcal{M}^{\infty}$ but there does not exist a nonzero polynomial $b$ such that $b\left(\mathcal{M}^{\prime}\right)^{\infty} \subseteq \mathcal{M}^{\infty}$. Since $\phi \in \mathcal{M}^{\prime}$, this contradicts that $\phi \in \mathcal{M}^{\infty, \ell}$.

CORollary 3.3. Suppose $(\operatorname{supp} \mu) \cap D$ is a uniqueness set for $\mathscr{H o l}(D)$. If $H_{\phi}^{\text {big }}$ is of finite rank, then $H_{\phi}^{\text {big }}=0$.

Proof. Theorem 3.2(3) implies the corollary. In fact, if $g \in L_{a}^{2} \cap L^{\infty}$, then $g(z)-$ $g(a) \in(z-a) L_{a}^{2}$ by [6, the proof in (1) of Theorem 5.4]. Thus $(g(z)-g(a)) /(z-a)$ belongs to $L_{a}^{2} \cap L^{\infty}$.

COROLLARY 3.4. Suppose $d \mu=r d r d \theta / \pi$. Let $D_{0}$ be an open subset of $D$ and $\mu=$ $\left\{f \in L^{2} ; f\right.$ is analytic on $\left.D_{0}\right\}$. Then $M$ is an invariant subspace and if $H_{\phi}^{\mu}$ is of finite rank then $H_{\phi}^{\mu}=0$.

Proof. It is easy to see that $\mathcal{M}^{\infty}$ satisfies Theorem 3.2(3).

COROLLARY 3.5. Suppose that if $H_{\phi}^{\mu}$ is of finite rank then $H_{\phi}^{\mu}=0$. If $\mathcal{M}^{\prime}$ is an invariant subspace which contains $\mathcal{M}$ properly, then the codimension of $\mathcal{M}$ in $\mathcal{M}^{\prime}$ is infinite or $\left(\mathcal{M}^{\prime}\right)^{\infty}=\mathcal{M}^{\infty}$.

Proof. If $\operatorname{dim} \mu^{\prime} / \mu<\infty$, as in the proof of Proposition 2.2, then there exists a nonzero polynomial $b$ such that $b \mathcal{M}^{\prime} \subseteq \mathcal{M}$. Hence $b\left(\mathcal{M}^{\prime}\right)^{\infty} \subseteq \mathcal{M}^{\infty}$. If $\left(\mathcal{M}^{\prime}\right) \neq \mathcal{M}^{\infty}$, by Theorem 3.2, this contradicts that if $H_{\phi}^{\mu}$ is of finite rank, then $H_{\phi}^{\mu}=0$.

4. General case. In this section, we assume that $d \mu=d \sigma(r) d \theta$ and $d \sigma([t, 1))>0$ for any $t>0$. Hence we can define the Fourier coefficients $\left\{\mathcal{M}_{j}\right\}_{j=-\infty}^{\infty}$ of $\mathcal{M}$. We assume $\mathcal{M}=\tilde{M}$, that is, $\mathcal{M}$ has the Fourier decomposition. 
THEOREM 4.1. Suppose $M$ is an invariant subspace which contains $z L_{a}^{2}$ and $\phi=$ $\sum_{j=-\infty}^{\infty} \phi_{j}(r) e^{i j \theta}$ is a function in $L^{\infty}$. Then $H_{\phi}^{\mu}$ is of finite rank $\leq \ell$ if and only if there exist complex numbers $b_{0}, \ldots, b_{\ell}$ such that $b_{\ell}=1$ and, for any integer $n$,

$$
\sum_{j=0}^{\ell} b_{j} r^{j} \phi_{n-j}(r) \in \mathcal{M}_{n}
$$

If $\ell$ is the minimum number of complex numbers $b_{1}, \ldots, b_{\ell}$ such that $\sum_{j=0}^{\ell} b_{j} r^{j} \phi_{n-j}(r)$ $\in \mathcal{M}_{n}$ for all $n$, then $H_{\phi}^{\mu}$ is of rank $\ell$.

Proof. If $H_{\phi}^{\mathcal{M}}$ is of rank $\leq \ell$, by Theorem 3.1 there exists a polynomial $b=\sum_{j=0}^{\ell} b_{j} z^{j}$ such that $b \phi \in \mathcal{M}$. Then

$$
\left(\sum_{j=-\infty}^{\infty} \phi_{j}(r) e^{i j \theta}\right)\left(\sum_{j=0}^{\ell} b_{j} r^{j} e^{i j \theta}\right)=\sum_{n=-\infty}^{\infty}\left(\sum_{j=0}^{\ell} \phi_{n-j}(r) b_{j} r^{j}\right) e^{i n \theta}
$$

and so $\sum_{j=0}^{\ell} b_{j} r^{j} \phi_{n-j}(r) \in \mathcal{M}_{n}$ for any $n$. The converse and the second statement are clear by Theorem 3.2.

COROLlaRY 4.2. Let $\phi=\phi_{t}(r) e^{i t \theta}$ for some integer $t$ in Theorem 4.1. Then $H_{\phi}^{\mu}$ is of finite rank $\leq \ell$ if and only if there exist complex numbers $b_{0}, \ldots, b_{\ell}$ such that $b_{\ell}=1$ and for $t \leq n \leq \ell+t, b_{n-t} r^{n-t} \phi_{t}(r) \in \mathcal{M}_{n}$.

Proof. Since $\phi_{j}(r)=0$ for $j \neq t$, if $n<t$ or $n>\ell+t$, then $\sum_{j=0}^{\ell} b_{j} r^{j} \phi_{n-j}(r)=0$. For $t \leq n \leq \ell+t, \sum_{j=0}^{\ell} b_{j} r^{j} \phi_{n-j}(r)=b_{n-t} r^{n-t} \phi_{t}(r)$, thus the corollary follows.

COROLLARY 4.3. Let $\phi=\sum_{j=1}^{\infty} a_{j} z^{j}+\sum_{j=0}^{\infty} a_{-j} \bar{z}^{j}$ in Theorem 4.1. Then $H_{\phi}^{\mu}$ is of rank $\leq \ell$ if and only if there exist complex numbers $b_{0}, \ldots, b_{\ell}$ such that $b_{\ell}=1$ and for any nonpositive integer $n \sum_{j=0}^{\ell} b_{j} a_{n-j} r^{2 j-n} \in \mathcal{M}_{n}$ and, for $0<n<\ell, \sum_{j=n}^{\ell} b_{j} a_{n-j} r^{2 j-n}$ $\in \mathcal{M}_{n}$.

Proof. If $n \geq \ell$ and $n \neq 0$, then

$$
\sum_{j=0}^{\ell} b_{j} r^{j} \phi_{n-j}(r)=\sum_{j=0}^{\ell} b_{j} a_{n-j} r^{j+n-j}=\left(\sum_{j=0}^{\ell} b_{j} a_{n-j}\right) r^{n}
$$

and hence $\sum_{j=0}^{\ell} b_{j} r^{j} \phi_{n-j}(r) \in \mathcal{M}_{n}$ because $z L_{a}^{2} \subseteq M$. Now Theorem 4.1 implies the corollary.

Theorem 4.1 does not give an exact relation between the rank of $H_{\phi}^{\mathcal{M}}$ and the number $\ell$ of complex numbers $b_{0}, \ldots, b_{\ell}$ such that $b_{\ell}=1$. However, we can show the following: if $H_{\phi}^{\mu}$ is of rank $\ell$, then there exist complex numbers $b_{0}, \ldots, b_{\ell}$ such that $b_{\ell}=1, \sum_{j=0}^{\ell} b_{j} r^{j} \phi_{n-j}(r) \in M_{n}$ for any $n$ and $b=\sum_{j=0}^{\ell} b_{j} z^{j}$ has just $\ell$ zeros in $D$. That is, if $\ell=1$, then $\left|b_{0}\right|<1$.

By Theorem 4.1, $H_{\phi}^{\mu}=0$ if and only if $\phi_{n} \in \mathcal{M}_{n}$ for any $n$ (i.e., $\phi \in \mathcal{M}$ ). Moreover, $H_{\phi}^{\mu}$ is of rank $\leq 1$ if and only if there exist complex numbers $\left(b_{0}, b_{1}\right) \neq(0,0)$ such that $b_{1}=1$ and $b_{0} \phi_{n}+b_{1} r \phi_{n-1} \in \mathcal{M}_{n}$ for any $n$. 
5. Big Hankel operator and $\mu \subseteq H^{2}$. In this section, we assume that $d \mu=d \sigma(r) d \theta$ and $d \sigma([t, 1))>0$ for any $t>0$. Hence we can define the Fourier coefficients $\left\{\mathcal{M}_{j}\right\}_{j=-k}^{\infty}$ of $\mathcal{M}$ and we assume $\mathcal{M}=\tilde{M}$. In this case, $H_{\phi}^{\mu}$ is close to $H_{\phi}^{\text {big }}$. Recall examples in Section 2, that is, $T_{\beta}, \bar{E}^{k}, Y(p)$, and $Y^{k}$.

COROLLARY 5.1. Suppose $M$ is an invariant subspace between $z L_{a}^{2}$ and $\mathbf{H}^{2}$, and $\phi=$ $\sum_{j=1}^{\infty} a_{j} z^{j}+\sum_{j=0}^{\infty} a_{-j} \bar{z}^{j}$. Then $H_{\phi}^{\mu}$ is of finite rank $\leq \ell$ if and only if $a_{-n}=0$ for $n>\ell$ and there exists complex numbers $b_{0}, \ldots, b_{\ell}$ such that $b_{\ell}=1$ and $\sum_{j=n}^{\ell} b_{j} a_{n-j} r^{2 j-n} \in M_{n}$ for $0 \leq n \leq \ell$ and $\sum_{j=0}^{\ell} b_{j} a_{n-j} r^{2 j-n}=0$ for $-\ell<n<0$.

Proof. Since $\mu \subseteq \mathbf{H}^{2}$, by Corollary $4.3 H_{\phi}^{\mu}$ is of finite rank $\leq \ell$ if and only if there exist complex numbers $b_{0}, \ldots, b_{\ell}$ such that $b_{\ell}=1$ and $\sum_{j=0}^{\ell} b_{j} a_{n-j} r^{2 j-n}=0$ for $n<0$ and $\sum_{j=n}^{\ell} b_{j} a_{n-j} r^{2 j-n} \in \mu_{n}$ for $0 \leq n \leq \ell$. If $\sum_{j=0}^{\ell} b_{j} a_{n-j} r^{2 j-n}=0$ for $n<0$, then $b_{j} a_{n-j}=0$ for $0 \leq j \leq \ell$ and $n<0$. Hence for each $j(0 \leq j \leq \ell), b_{j} a_{-t}=0$ if $t>j$. Thus $a_{-t}=0$ if $t>\ell$.

Proposition 5.2. Suppose $\mathcal{M}$ is an invariant subspace between $z L_{a}^{2}$ and $e^{-i k \theta} \mathbf{H}^{2}$ where $k \geq 0$, and $\phi=\sum_{j=0}^{\infty} \phi_{-j}(r) e^{-i j \theta}$ is a function in $L^{\infty}$. Then $H_{\phi}^{\mu}$ is of finite rank $\leq \ell$ if and only if

$$
\phi(z)=\frac{\sum_{j=-k}^{\ell} \psi_{j}(r) e^{i j \theta}}{\sum_{j=0}^{\ell} b_{j} r^{j} e^{i j \theta}},
$$

where $\psi_{n}=\sum_{j=0}^{\ell} b_{j} r^{j} \phi_{n-j} \in M_{n}$, for $-k \leq n \leq \ell$, and $\left(b_{0}, \ldots, b_{\ell}\right) \in \mathbb{C}^{\ell}$.

Proof. Note that $\mu \subseteq e^{-i k \theta} \mathbf{H}^{2}$ and $\phi_{j}(r)=0$ for $j>0$. If $H_{\phi}^{\mu}$ is of finite rank $\leq \ell$, then, by Theorem 4.1,

$$
\left(\sum_{j=0}^{\ell} b_{j} r^{j} e^{i j \theta}\right)\left(\sum_{j=0}^{\infty} \phi_{-j}(r) e^{-i j \theta}\right)=\sum_{n=-k}^{\ell} \psi_{n}(r) e^{i n \theta}
$$

and $\psi_{n}=\sum_{j=0}^{\ell} b_{j} r^{j} \phi_{n-j} \in M_{n}$ for $-k \leq n \leq \ell$. The converse is also a result of Theorem 3.1.

Corollary 5.3. Suppose $\mu$ is an invariant subspace in Proposition 5.2. If $\phi=$ $\phi_{+}+\phi_{-}=\sum_{j=1}^{\infty} a_{j} z^{j}+\sum_{j=0}^{\infty} a_{-j} \bar{z}^{j}$ and $\phi_{-} \in L^{\infty}$, then $H_{\phi}^{\mu}$ is of finite rank $\leq \ell$ if and only if

$$
\phi(z)=\phi_{+}+\frac{\sum_{j=-k}^{\ell} \psi_{j}(r) e^{i j \theta}}{\sum_{j=0}^{\ell} b_{j} r^{j} e^{i j \theta}}
$$

where $\psi_{n}=\sum_{j=0}^{\ell} b_{j} a_{n-j} r^{j+|n-j|} \in \mu_{n}$, for $-k \leq n \leq \ell$, and $\left(b_{0}, \ldots, b_{\ell}\right) \in \mathbb{C}^{\ell}$. If $\left(b_{0}, \ldots, b_{\ell}\right)=(0, \ldots, 0)$, then $\psi_{n}=0$ and so $\phi=\phi_{+}$.

THEOREM 5.4. Suppose $M$ is an invariant subspace between $z L_{a}^{2}$ and $e^{-i k \theta} \mathbf{H}^{2}$ where $k \geq 0$, and $\phi=\sum_{j=1}^{\infty} \phi_{-j}(r) e^{i j \theta}$ is a function in $L^{\infty}$.

(1) If $\mu_{j} \cap r^{j+1} \mathscr{L}^{2}=\{0\}$ for any $j \geq 0$, then there does not exist any finite rank $H_{\phi}^{\mu}$ except $H_{\phi}^{\mu}=0$.

(2) If there does not exist any finite rank $H_{\phi}^{\mu}$ except $H_{\phi}^{\mu}=0$, then $\mu_{-(k-j)} \cap r^{j+1} \mathscr{L}^{\infty}=$ $\{0\}$ for any $j \geq 0$. 
Proof. (1) If $H_{\phi}^{\mu}$ is of finite rank $\ell$, by Proposition 5.2,

$$
\psi_{n}=\sum_{j=n}^{\ell} b_{j} r^{j} \phi_{n-j} \in M_{n},
$$

for $0 \leq n \leq \ell$ because $\phi_{n-j}(r)=0$ for $0 \leq j \leq n-1$. We may assume $b_{\ell}=1$. As $n=\ell-1, r^{\ell} \phi_{-1}(r) \in M_{\ell-1}$. Since $M_{\ell-1} \cap r^{\ell} \mathscr{L}^{2}=\{0\}, \phi_{-1}(r)=0$. As $n=\ell-2$,

$$
b_{\ell-1} r^{\ell-1} \phi_{-1}(r)+r^{\ell} \phi_{-2}(r) \in M_{\ell-2} \text {. }
$$

Since $M_{\ell-2} \cap r^{\ell-1} \mathscr{L}^{2}=\{0\}$ and $\phi_{-1}(r)=0, \phi_{-2}(r)=0$. we can get $\phi_{-j}(r)=0$ for $j \leq \ell$. In Proposition 5.2, $\psi_{n}=0$ for $0 \leq n \leq \ell$ and so $\phi \equiv 0$.

(2) If $r^{j+1} g \in \mathcal{M}_{-(k-j)} \cap r^{j+1} \mathscr{L}^{\infty}$, then put $\phi=g e^{-i(k+1) \theta}$. If $g \neq 0$ then $\phi \notin \mathcal{M}$ and

$$
z^{j+1} \phi=r^{j+1} g e^{-i(k-j) \theta} \in \mathcal{M}_{-(k-j)} e^{-i(k-j) \theta} .
$$

Since $\mathcal{M}$ has the Fourier decomposition, $\mathcal{M}_{j} e^{i j \theta} \subseteq \mathcal{M}$ and so $z^{j+1} \phi \in \mathcal{M}$. Theorem 3.1 gives a contradiction.

We will apply results in this section to Example 2.4 in Section 2.

EXAMPLE 5.5. (i) Suppose $\mu=T_{\beta}(0 \leq \beta<1)$.

(1) When $\phi=\sum_{j=1}^{\infty} \phi_{-j}(r) e^{-i j \theta}$ is a function in $L^{\infty}$, there does not exist any finite rank $H_{\phi}^{\mu}$ except $H_{\phi}^{\mu}=0$ if and only if $\beta=0$.

(2) When $\phi=\sum_{j=0}^{\infty} a_{j} z^{j}+\sum_{j=1}^{\infty} a_{-j} \bar{z}^{j}$ is a function in $L^{\infty}$, there does not exist any finite rank $H_{\phi}^{\mu}$ except $H_{\phi}^{\mu}=0$ if and only if $\beta=0$.

Proof. Recall that $T_{\beta}=\sum_{j=0}^{\infty} \oplus\left(T_{\beta}\right)_{j} e^{i j \theta}$ and $\left(T_{\beta}\right)_{j}=\operatorname{span}\left\{r^{j} p_{j}\left(r^{2}\right) ; p_{j}\right.$ is a polynomial of degree at most $\beta j / 1-\beta$.

(1) If $\beta=0$, then $\left(T_{\beta}\right)_{j} \cap r^{j+1} \mathscr{L}^{2}=\{0\}$ for any $j \geq 0$ and if $\beta \neq 0$, then $\left(T_{\beta}\right)_{j} \cap$ $r^{j+1} \mathscr{L}^{\infty} \neq\{0\}$ for enough large $j$. Theorem 5.4 implies (1).

(2) If $\beta \neq 0$, then there exists $n$ such that $1-\beta \leq \beta(n-1)$. Hence $\left(T_{\beta}\right)_{n-1} \ni r^{n+1}$. Suppose $\phi=\bar{z}$, then $z^{n} \phi=r^{n+1} e^{i(n-1) \theta}$ and so $z^{n} \phi \in\left(T_{\beta}\right)_{n-1} e^{i(n-1) \theta} \subset T_{\beta}$. By Theorem 3.1, $H_{\phi}^{\mu}$ is of rank $\leq n$ and $H_{\phi}^{\mu} \neq 0$.

(ii) Suppose $\mathcal{M}=\bar{E}^{m}(0 \leq m<\infty)$.

(1) When $\phi=\sum_{j=1}^{\infty} \phi_{-j}(r) e^{-i j \theta}$, there does not exist any finite rank $H_{\phi}^{\mu}$ except $H_{\phi}^{\mu}=0$ if and only if $m=0$.

(2) When $\phi=\sum_{j=0}^{\infty} a_{j} z^{j}+\sum_{j=1}^{\infty} a_{-j} \bar{z}^{j}$ is a function in $L^{\infty}$, there does not exist any finite rank $H_{\phi}^{\mu}$ except $H_{\phi}^{\mu}=0$ if and only if $m=0$ or 1 .

Proof. We recall that $(\bar{E})^{m}=\sum_{j=0}^{\infty} \oplus\left(\bar{E}^{m}\right)_{j} e^{i j \theta}$ and $\left(\bar{E}^{m}\right)_{j}=\operatorname{span}\left\{r^{j}, \ldots, r^{j+2 m}\right\}$.

(1) If $m=0$, then $\left(\bar{E}^{m}\right)_{j} \cap r^{j+1} \mathscr{L}^{2}=\{0\}$ for any $j \geq 0$ and if $m \neq 0$, then $\left(\bar{E}^{m}\right)_{j} \cap$ $r^{j+1} \mathscr{L}^{\infty} \neq\{0\}$ for any $j \geq 0$. Theorem 5.4 implies (1).

(2) If $m=0$, by (1) there does not exist any finite rank $H_{\phi}^{\mu}$ except $H_{\phi}^{\mu}=0$. If $m=1$, then $\left(E^{m}\right)_{n}=\operatorname{span}\left\{r^{n}, r^{n+2}\right\}$ for $n \geq 0$. When $H_{\phi}^{M}$ is of finite rank $\ell$, by Corollary 5.1, $a_{-n}=0$ for $n>\ell$ and if $0 \leq n \leq \ell$,

$$
\sum_{j=n}^{\ell} b_{j} a_{n-j} r^{2 j-n}=c r^{n}+d r^{n+2}
$$


for complex constants $c, d$. Hence, for $0 \leq n \leq \ell$,

$$
b_{j} a_{n-j}=0 \text { for } n+2 \leq j \leq \ell .
$$

Since $b_{\ell}=1, a_{n-\ell}=0$ for $0 \leq n \leq \ell$ and so $a_{-j}=0$ for $0 \leq j \leq \ell$. When $m \geq 2$, if $\phi=\bar{z}$, then $z \phi=r^{2} \in\left(\bar{E}^{m}\right)_{0}=\operatorname{span}\left\{1, r^{2}, \ldots, r^{2 m}\right\}$ and $z \phi \in \bar{E}^{m}$ because $\left(\bar{E}^{m}\right)_{0} \subset \bar{E}^{m}$. However $H_{\phi}^{\mu} \neq 0$.

(iii) Suppose $M=Y^{k}$.

(1) When $\phi=\sum_{j=1}^{\infty} \phi_{-j}(r) e^{-i j \theta}$, there does not exist any finite rank $H_{\phi}^{\mu}$ except $H_{\phi}^{M}=0$ if and only if $k=0$.

(2) When $\phi=\phi_{+}+\phi_{-}=\sum_{j=0}^{\infty} a_{j} z^{j}+\sum_{j=1}^{\infty} a_{-j} \bar{z}^{j}$ and $\phi_{+}$are functions in $L^{\infty}$, there does not exist any finite rank $H_{\phi}^{\mu}$ except $H_{\phi}^{\mu}=0$ if and only if $k=0$.

Proof. Since $H_{\phi}^{\mu}=H_{\phi_{-}}^{\mu}$, it is sufficient to prove (1). We recall that $Y^{k}=$ $\sum_{j=-k}^{\infty} \oplus\left(Y^{k}\right)_{j} e^{i j \theta}$, where $Y_{0}^{k}=\operatorname{span}\left\{1, r^{2}, \ldots, r^{2 k}\right\}$ and $\left(Y^{k}\right)_{j}=r^{j}\left(Y^{k}\right)_{0}$ for $j \geq 0$, and $\left(Y^{k}\right)_{-j}=\operatorname{span}\left\{r^{2 \ell-j}, j \leq \ell \leq k\right\}$ for $1 \leq j \leq k$. If $k=0$, then $Y^{k}=L_{a}^{2}$. If $k \geq 1$, $\left(Y^{k}\right)_{-k}=\operatorname{span}\left\{r^{k}\right\}$. Theorem 5.4(2) implies that there exists a nonzero finite rank $H_{\phi}^{\mu}$.

6. Small Hankel operator and $\mu \supseteq \mathbf{H}^{2}$. In this section, we assume that $d \mu=$ $d \sigma(r) d \theta$ and $d \sigma([t, 1))>0$ for any $t>0$. Hence we can define the Fourier coefficients $\left\{\mathcal{M}_{j}\right\}_{j=-\infty}^{\infty}$ of $\mathcal{M}$. In this case, $H_{\phi}^{\mu}$ is close to $H_{\phi}^{\text {small }}$ and far from $H_{\phi}^{\text {big }}$. Note that if $\mathcal{M}^{\prime}$ is an invariant subspace and $\mathcal{M}^{\prime} \subseteq e^{i \theta} \mathbf{H}^{2}$, then $\mathcal{M}=\left(\overline{\mathcal{M}}^{\prime}\right)^{\perp}$ is an invariant subspace and $\mu \supseteq e^{i \theta} \mathbf{H}^{2}$.

Proposition 6.1. Suppose $\mathcal{M}$ is an invariant subspace which contains $e^{i k \theta} \mathbf{H}^{2}$ for some nonnegative integer $k$. If $\mu \neq L^{2}$, there exists at least a nonzero finite rank $H_{\phi}^{\mu}$.

Proof. If $\bar{z}^{n} \in M$ for all $n \geq 1$, then $z^{\ell} \bar{z}^{n} \in \mathcal{M}$ for all $\ell \geq 1$ because $z M \subseteq M$. Let $\mathscr{E}$ be the closed linear span of $\left\{z^{\ell} \bar{z}^{n} ; n \geq 1, \ell \geq 0\right\}$, then $\mathscr{E} \subseteq \mathcal{M}$ and $g_{\mathscr{E}}^{\mathscr{E}} \subseteq \mathscr{E}$ for arbitrary polynomial $g$ of $z$ and $\bar{z}$. It is well known that $\mathscr{E}=L^{2}$. This contradiction implies that there exists at least $n$ such that $\bar{z}^{n} \notin \mathcal{M}$ and $n \geq 1$. If $\phi=\bar{z}^{n}$, then $z^{n+k} \phi \in \mathcal{M}$. Then $H_{\phi}^{\mu} \neq 0$ but $H_{\phi}^{\mu}$ is of finite rank $\leq n+k$, by Theorem 3.1.

Proposition 6.2. Suppose $\mathcal{M}$ is an invariant subspace which contains $e^{i k \theta} \mathbf{H}^{2}$ for some nonnegative integer $k$. The following statements are valid.

(1) If $\phi=\sum_{j=-\infty}^{\infty} \phi_{j}(r) e^{i j \theta}$ is a function in $L^{\infty}$, then there exists a function $\phi^{\prime}$ in $L^{2}$ such that $\phi^{\prime}=\sum_{j=0}^{k-1} \phi_{j}(r) e^{i j \theta}+\sum_{j=1}^{\infty} \phi_{-j}(r) e^{-i j \theta}$ and $H_{\phi^{\prime}}^{\mu}=H_{\phi}^{\mu}$.

(2) If $\phi=\sum_{j=k}^{\infty} \phi_{j}(r) e^{i j \theta}$ is a function in $L^{\infty}$, then $H_{\phi}^{\mu}=0$.

(3) If $\phi=\sum_{j=-\ell}^{\infty} \phi_{j}(r) e^{i j \theta}$ is a function in $L^{\infty}$, then $H_{\phi}^{\mu}$ is of rank $\leq \ell+k<\infty$. Conversely, if one of (1) or (2) is valid, then $M$ contains $e^{i k \theta} \mathbf{H}^{2}$.

Proof. Both (1) and (2) are clear because $\mu \supseteq e^{i k \theta} \mathbf{H}^{2}$. (3) is a result of Theorem 3.1. The converse is also clear.

We will consider Example 2.4 in Section 2.

EXAMPLE 6.3. (ii) Suppose $\mathcal{M}=\left(E^{k}\right)^{\perp}(0 \leq k<\infty)$ and $\phi=\sum_{j=-\infty}^{\infty} \phi_{j}(r) e^{i j \theta}$ is a function in $L^{\infty}$. 
(1) $H_{\phi}^{\mu}=0$ if and only if

$$
\int_{0}^{1} \phi_{-j}(r) r^{j+2 t} d \sigma=0 \quad(j \geq 0,0 \leq t \leq k) .
$$

(2) $H_{\phi}^{\mathcal{M}}$ is of rank $\leq 1$ if and only if there exist complex numbers $\left(b_{0}, b_{1}\right) \neq(0,0)$ such that

$$
b_{0} \int_{0}^{1} \phi_{-j}(r) r^{j+2 t} d \sigma=-b_{1} \int_{0}^{1} \phi_{-j-1}(r) r^{j+2 t+1} d \sigma
$$

for $j \geq 0,0 \leq t \leq k$.

(3) Suppose $d \sigma=r d r / 2 \pi$. When $\phi=\sum_{j=0}^{\infty} a_{j} z^{j}+\sum_{j=1}^{\infty} a_{-j} \bar{z}^{j}$, if $H_{\phi}^{\mu}$ is of rank $\leq 1$, then $H_{\phi}^{\mu}=0$.

Proof. From the remark in the last part of Section 4, (1) and (2) follows. (3) By (2), $H_{\phi}^{\mu}$ is of rank $\leq 1$ if and only if there exist complex numbers $\left(b_{0}, b_{1}\right) \neq(0,0)$ such that

$$
b_{0} a_{-j} \frac{1}{2 j+2 t+1}=-b_{1} a_{-j-1} \frac{1}{2 j+2 t+3}
$$

for $j \geq 0,0 \leq t \leq k$. When $k \neq 0$, for each $j$, as $t=0$,

$$
\begin{aligned}
& b_{0} a_{-j} \frac{1}{2 j+1}=-b_{1} a_{-j-1} \frac{1}{2 j+3}, \\
& b_{0} a_{-j} \frac{1}{2 j+3}=-b_{1} a_{-j-1} \frac{1}{2 j+5} .
\end{aligned}
$$

This implies that $a_{-j}=a_{-j-1}=0$, for $j \geq 0$, and so $\phi=\sum_{j=1}^{\infty} a_{j} z^{j}$. When $k=0$, Corollary 3.3 implies (3)

(iv) Suppose $\mu=\bar{q} \mathbf{H}^{2}$ for some unimodular function $q$ in $\mathbf{H}^{2}$ and $\phi$ is a function in $L^{\infty} . H_{\phi}^{M}$ is of finite rank $\ell$ if and only if

$$
\phi=\bar{q} \sum_{j=-\ell}^{\infty} \psi_{j}(r) e^{i j \theta},
$$

where $\psi_{-\ell}(r) \neq 0$.

Proof. If $\phi=\bar{q} \sum_{j=-\ell}^{\infty} \psi_{j}(r) e^{i j \theta}$, then $z^{\ell} \phi \in \mathcal{M}$ and so, by Theorem 3.1, $H_{\phi}^{\mu}$ is of finite rank $\leq \ell$. Since $\psi_{-\ell}(r) \neq 0, b \phi \notin \mathcal{M}$ for any polynomial $b$ of degree $\leq \ell-1$ and so $H_{\phi}^{\mu}$ is of finite rank $\ell$. The converse is clear.

(v) Suppose $\mathcal{M}=\mathbf{H}^{2} \oplus S e^{-i \theta}$ and $S$ is a closed subspace in $\mathscr{L}^{2}$. Let $\phi=\sum_{j=-\infty}^{\infty} \phi_{j}(r) e^{i j \theta}$ be a function in $L^{\infty}$. By Theorems 3.1 and $4.1, H_{\phi}^{\mu}$ is of finite rank $\leq \ell$ if and only if $\phi_{j}(r)=0$ for $j \leq-(\ell+2)$ and there exist complex numbers $b_{0}, \ldots, b_{\ell}$ such that $b_{\ell}=1$,

$$
\begin{gathered}
\sum_{j=0}^{\ell} b_{j} r^{j} \phi_{n-j}(r)=0 \text { for }-(\ell+1) \leq n<-1, \\
\sum_{j=0}^{\ell} b_{j} r^{j} \phi_{-1-j}(r) \in S .
\end{gathered}
$$


7. Restricted shift operator and $\mu \subseteq L_{a}^{2}$. In this section, we assume $\mu=r d r d \theta / \pi$ for simplicity. Let $\mathcal{M}$ be an invariant subspace in $L_{a}^{2}$ and $\mathscr{K}=L_{a}^{2} \ominus \mu$. For $\phi$ in $L_{a}^{\infty}=$ $L_{a}^{2} \cap L^{\infty}$,

$$
S_{\phi}^{\mathscr{K}} f=\left(I-P^{\mathscr{K}}\right)(\phi f) \quad(f \in \mathscr{K}),
$$

where $P^{\mathscr{K}}$ is the orthogonal projection from $L_{a}^{2}$ to $\mathscr{K} . S_{z}^{\mathscr{K}}$ is called a restricted shift operator. For any $\phi$ in $L_{a}^{\infty}, S_{\phi}^{\mathscr{K}}$ commutes with $S_{z}^{\mathscr{K}}$. We do not know whether if the bounded linear operator $T$ on $\mathscr{K}$ commutes with $S_{z}^{\mathscr{K}}$, then $T=S_{\phi}^{\mathscr{K}}$ for some $\phi$ in $L_{a}^{\infty}$. If $T S_{z}^{\mathscr{K}}=S_{z}^{\mathscr{K}} T$ and $\phi=T P^{\mathscr{K}} 1$ is bounded, then it is easy to see that $T=S_{\phi}^{\mathscr{K}}$ (cf. [5, page 784]). In the Hardy space instead of the Bergman space, Sarason [8] showed that this is true without any condition and $\|T\|=\|\phi\|_{\infty}$.

We can define the Hankel operator $H_{\phi}^{\mu}$ as in the introduction. However $H_{\phi}^{\mu}$ is not an intermediate Hankel operator. It is not so difficult to see the following: when $\mathscr{K}=$ $L_{a}^{2} \ominus \mathcal{M}$ and $\phi$ in $L_{a}^{\infty}$,

$$
\left\|H_{\phi}^{\mu}\right\|=\left\|S_{\phi}^{\mathscr{K}}\right\| .
$$

This is known for the Hardy space. In fact, for $f$ in $L_{a}^{2}$,

$$
H_{\phi}^{\mu} f=\left(I-P^{\mu}\right) \phi f=P^{\mathscr{K}} \phi P^{\mathscr{K}} f
$$

and so $H_{\phi}^{\mu} f=S_{\phi}^{\mathscr{K}} P^{\mathscr{K}} f$ for $f$ in $L_{a}^{2}$. Hence $H_{\phi}^{\mu}$ is of finite rank $n$ if and only if $S_{\phi}^{\mathscr{K}}$ is of finite rank $n$. It is easy to see that $S_{\phi}^{\mathscr{K}}$ is of finite rank $\ell \leq n$ if and only if there exists an analytic polynomial $p$ of degree $\ell \leq n$ such that $p(\phi) \in \mathcal{M}^{\infty}$. When $\phi$ is in $L^{\infty}$, Theorems 3.1 and 4.1 are true for $H_{\phi}^{\mu}$.

Suppose $\phi$ is a function in $L_{a}^{\infty}$.

(1) $L_{a}^{2} \supseteq \operatorname{ker} H_{\phi}^{\mu} \supseteq \mu$.

(2) When the common zero set $Z(\mathcal{M})$ of $\mathcal{M}$ in $D$ is empty, if $H_{\phi}^{\mu}$ is of finite rank then $H_{\phi}^{\mu}=0$. This is a result of (1) and Proposition 2.1.

(3) If $Z(\mathcal{M})$ is not empty, there exists a nonzero finite rank $H_{\phi}^{\mathcal{M}}$.

ACKNOWLEDGEMENT. This research was partially supported by Grant-in-Aid for scientific research, Ministry of Education.

\section{REFERENCES}

[1] S. Axler and P. Bourdon, Finite-codimensional invariant subspaces of Bergman spaces, Trans. Amer. Math. Soc. 306 (1988), no. 2, 805-817. MR 89f:46051. Zbl 658.47011.

[2] S. Janson and R. Rochberg, Intermediate Hankel operators on the Bergman space, J. Operator Theory 29 (1993), no. 1, 137-155. MR 95b:47026. Zbl 898.47015.

[3] T. Nakazi, Invariant subspaces of weak-* Dirichlet algebras, Pacific J. Math. 69 (1977), no. 1, 151-167. MR 58\#12384. Zbl 342.46044.

[4] _ Extended weak-* Dirichlet algebras, Pacific J. Math. 81 (1979), no. 2, 493-513. MR 82a:46055. Zbl 407.46047.

[5] _ Commuting dilations and uniform algebras, Canad. J. Math. 42 (1990), no. 5, 776789. MR 92b:46080. Zbl 762.46056.

[6] T. Nakazi and M. Yamada, Riesz's functions in weighted Hardy and Bergman spaces, Canad. J. Math. 48 (1996), no. 5, 930-945. MR 98c:46049. Zbl 862.46013.

[7] L. Z. Peng, R. Rochberg, and Z. Wu, Orthogonal polynomials and middle Hankel operators on Bergman spaces, Studia Math. 102 (1992), no. 1, 57-75. MR 93b:47054. Zbl 809.30008. 
[8] D. Sarason, Generalized interpolation in $H^{\infty}$, Trans. Amer. Math. Soc. 127 (1967), 179203. MR 34\#8193. Zbl 145.39303.

[9] E. Strouse, Finite rank intermediate Hankel operators, Arch. Math. (Basel) 67 (1996), no. 2, 142-149. MR 97i:47047. Zbl 905.47014.

[10] J. L. M. Wang and Z. Wu, Minimum solution of $\bar{\partial}^{k+1}$ and middle Hankel operators, J. Funct. Anal. 118 (1993), no. 1, 167-187. MR 94j:47042. Zbl 801.47017.

[11] N. Young, An Introduction to Hilbert Space, Cambridge Mathematical Textbooks. Cambridge University Press, Cambridge, New York, 1988. MR 90e:46001. Zbl 645.46024.

[12] K. Zhu, Operator Theory in Function Spaces, Monographs and Textbooks in Pure and Applied Mathematics, vol. 139, Marcel Dekker, Inc., New York, 1990. MR 92c:47031. Zbl 706.47019.

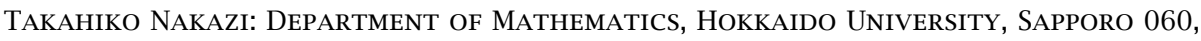
JAPAN

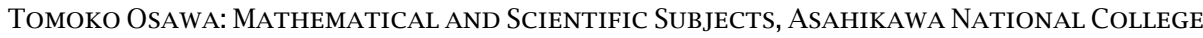
OF TECHNOLOGY, ASAHIKAWA 071, JAPAN 


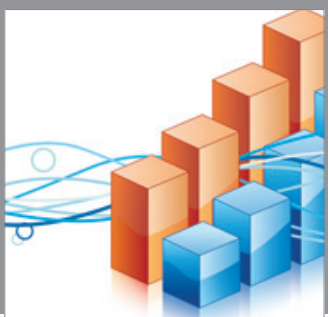

Advances in

Operations Research

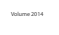

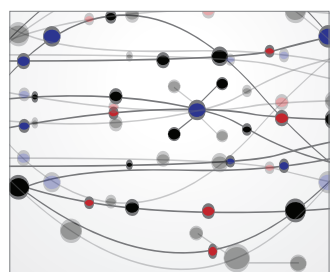

\section{The Scientific} World Journal
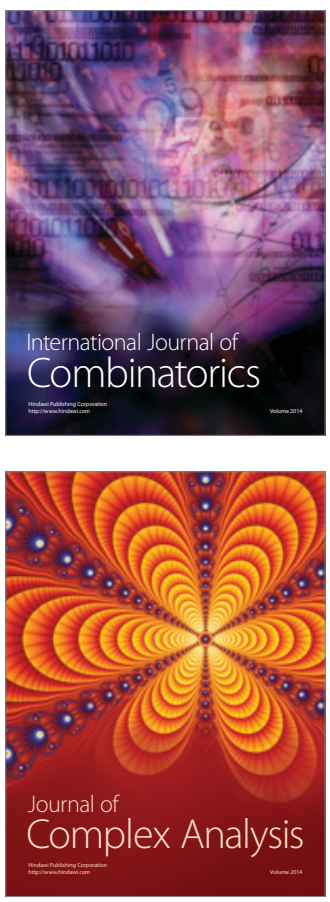

International Journal of

Mathematics and

Mathematical

Sciences
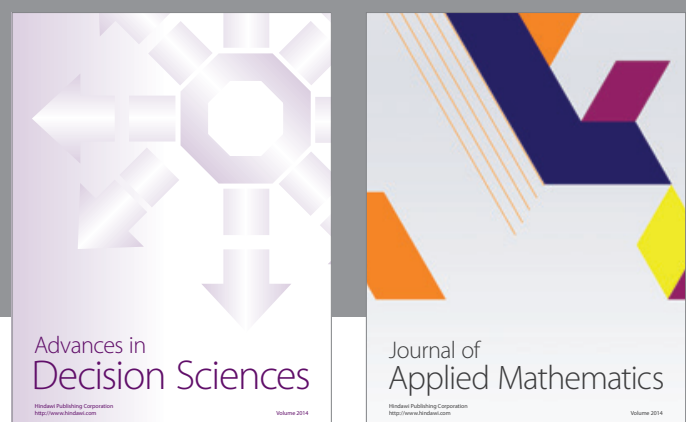

Journal of

Applied Mathematics
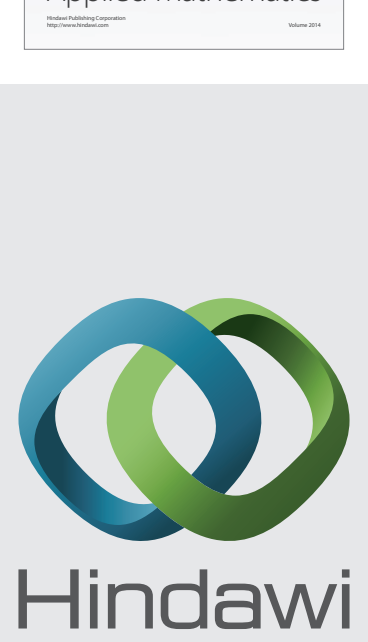

Submit your manuscripts at http://www.hindawi.com
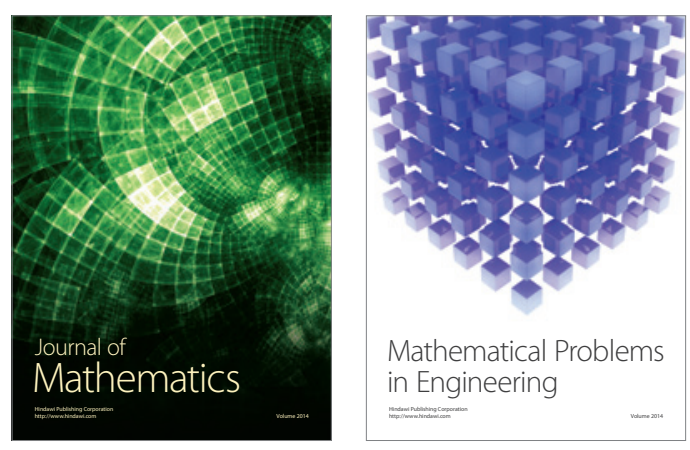

Mathematical Problems in Engineering
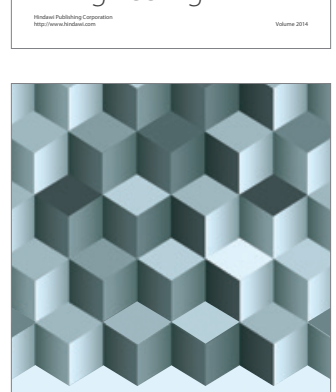

Journal of

Function Spaces
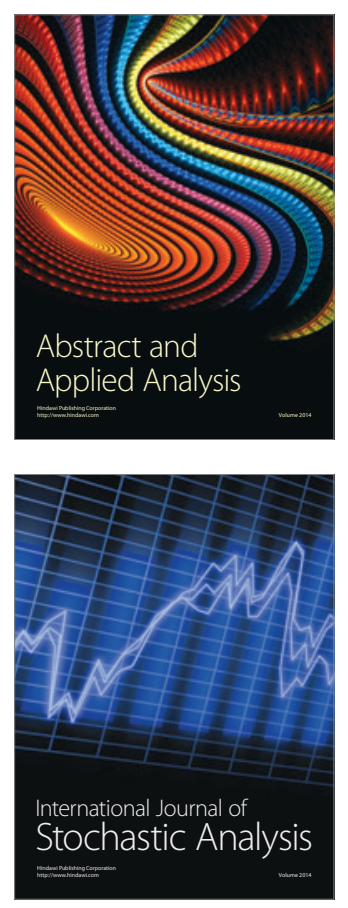

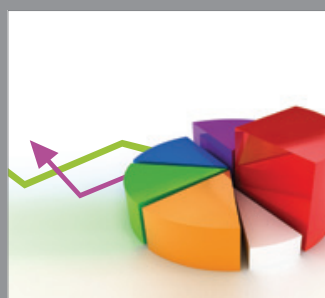

ournal of

Probability and Statistics

Promensencen
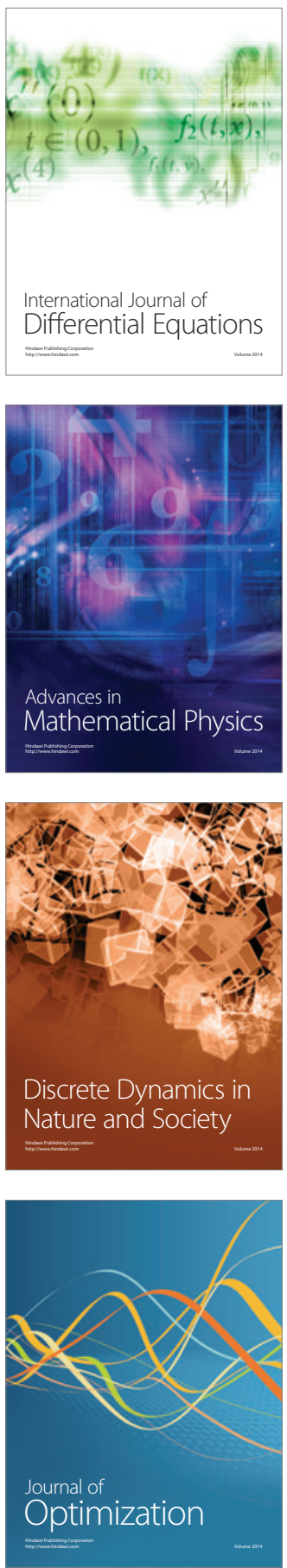